\title{
Identification of Inflating Pressure for a Diseased Artery During Stenting: FE Analysis
}

\author{
Hashim V ${ }^{1}$, Resmi SL ${ }^{2}$ Dileep PN PN $^{3}$ Fasin Ahammad \\ ${ }^{1}$ Associate Professor, Dept.of Mechanical Engineering, TKM College of Engineering, Kollam, Kerala, India. \\ (corresponding author) \\ ${ }^{2}$ Assistant Professor, Dept. of Mechanical Engineering, TKM College of Engineering, Kerala, India. \\ ${ }^{3}$ Professor, Dept. of Mechanical Engineering, TKM College of Engineering, Kerala, India. \\ ${ }^{4}$ UG Scholar, Dept. of Mechanical Engineering, TKM College of Engineering, Kerala, India.
}

\begin{abstract}
In coronary angioplasty a tubular stent is inserted in to a blocked artery and expanded at the blocked site to reinstate the blood flow. This treatment method has got wide acceptance since it is comparatively simple and fast curing. Atherosclerotic plaques are heterogeneous material with different mechanical behavior related to their composition and boundary conditions. It is not possible to conduct an in-vitro experiment to analyze the expansion behavior of a stent within the artery. However finite element method is a suitable tool widely used for this purpose. This paper study the expansion behavior of slotted tube stent in different cases (only stent, stent with balloon and stent within artery) using computational methods. The variation in stress distribution and expansion with respect to inflating pressure were compared. Significant difference was observed in the expansion behavior of stent with and without artery. An average of $10 \%$ reduction in the stress and $45 \%$ reduction in strain was noticed as stent expands inside the stenosed artery. Also the optimum pressure for the exact opening of a diseased artery was evaluated and observed that it varies with percentage of block and nature of plaque. An optimum pressure of $0.58 \mathrm{MPa}$ was identified for a diseased artery with $20.42 \%$ block to expand to its original diameter of $4 \mathrm{~mm}$.
\end{abstract}

Keywords: Atherosclerosis; Coronary stent; Expansion characteristics; Finite element analysis; Inflating pressure; Stenosed artery.

\section{INTRODUCTION}

Coronary heart disease is the major cause of death worldwide and its rate increases yearly. Angioplasty with stent is highly preferred therapy to treat coronary heart disease. In this non surgical procedure a coronary stent (tubular structure with specific geometry and material) with balloon is deployed to the blocked artery using a stent delivery system and the stent is expanded by inflating the balloon. Self expandable and balloon expandable are two different types of stents. Balloon expandable stents perform better than self expandable stents because its expansion can be controlled during deployment. After expansion, plastically deformed stent will act as a permanent scaffold to keep the blood vessel open and the blood flow reinstates. Stenting is popular in the treatment of coronary artery disease due to its simplicity and fast curing procedure. In the last four decades computational analysis have developed to such a level that any complicated non linear problem can be modeled and solved. Finite element method (FEM) is a suitable and valid tool to study the characteristics of stent during deployment [1]. Design analysis proved that both geometries of strut ring and strut connector have influence on its mechanical behavior. Many works had been undergone in this area and new design of stents are developed with better performance. However restenosis and thrombosis are some of the problems commonly seen in most of the patients after years. Hashim et.al. investigated the influence of design patterns on the expansion behavior of different models and found that number of strut connector will reduce the expansion and increase the stress concentration [2]. Frederique Etava et al. performed the analysis to compare the mechanical characteristics of two different types of stents: tubular stents and coil stents and established that tubular has better rigidity and coil stent has better flexibility [3]. Hyre MR et al. investigated the matching of stent to inflating balloon, artery and occlusion size and found that $10 \%$ increase in balloon overhang results in $4 \%$ increase in the maximum end flare and $39 \%$ change in peak arterial stress [4]. Francesco Migliavacca et al. used FEM to understand the effects of different geometrical parameters (thickness, metal-to-artery surface ratio, longitudinal and radial cut lengths) of a typical diamond-shaped coronary stent. The impact of stent pattern on restenosis rate was investigated using stress distribution in the arteries by S.N. David Chua et al. observed how the pressure on the balloon was transferred to the stent. The interaction of balloon and slotted tube stent discloses that higher inflating pressure is one of the major reasons for artery injury and instent restenosis [5], [6], [7]. N. Eshghi.et al. has investigated the expansion characteristics of a stent as it is implanted in an artery containing plaque, to get more realistic results [8]. Numerical analysis of stent crimping and implantation process in a diseased artery with balloon was performed by Jakub Bukala et.al and observed that the stent over sizing may lead to high intramural wall stress[9]. Stent deployment on a patient derived model with a three folded balloon was conducted by Houman to get optimum modeling strategy with accurate deployment of a stent in a realistic geometry of an atherosclerotic human coronary artery. It is found that application of pressure with constraints on expansion 
is an efficient method to predict the stress strain in the artery [10].

From the above discussions it is evident that the severity of atherosclerotic plaques to be assessed before undergoing stent deployment and the optimum inflating pressure is to be predicted based on the percentage of block, type of plaque and the design of stent. This can be approximated by reconstructing an accurate geometry of the diseased artery and stent models. In this work expansion characteristics of the stent within the artery are studied using FE method. FE analysis of stent deployment with and without artery was compared and stent expansion with variable input pressures was investigated with respect to stress and expansion.

\section{FE MODEL OF STENT SYSTEM}

The study involves FE analysis on expansion of balloon expandable artery stent inside a plaque artery system. A slotted tubular type commercially available Palmaz-schatz stent was modeled using SOLID WORKS software and the expansion process was analyzed in ABAQUS CAE 6.13. The geometry of the stent was reproduced as given in the literature [7]. The length of the stent was $10 \mathrm{~mm}$ with an inner and outer diameter of $2.90 \mathrm{~mm}$ and $3 \mathrm{~mm}$ respectively. Due to the symmetry of the stent along the circumferential axis, one forth of the circumference with full length as shown in Fig.1 was used to simulate the expansion process.

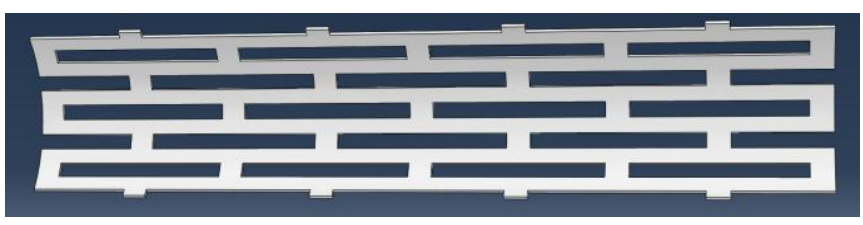

Fig. 1: 3D model of palmaz stent

An elasto plastic material was assumed for the stent material and its properties were chosen to approximately represent the behavior of Stainless Steel 304. (Young's modulus=193GPa; Density $=7.86 \mathrm{e}-{ }^{6} \mathrm{~kg} / \mathrm{m}^{3}$; Poisson ratio $=0.27$; Yield strength $=$ 207MPa). The plastic strain data were also extracted from literature [11] to mimic the plastic deformation experienced by the stent during expansion. The balloon as a medium to expand was modeled as quarter cylinder of $12 \mathrm{~mm}$ in length. outer diameter $2.9 \mathrm{~mm}$ and thickness $0.1 \mathrm{~mm}$. A polyurethane rubber type material was used to represent the balloon as described by a hyper elastic Mooney-Rivlin strain energy function given in (1).

$$
W=C_{10}\left(I_{1}-3\right)+C_{01}\left(I_{2}-3\right)+1 / D_{1}(J-3)
$$

Where $I_{1}$ and $I_{2}$ are the first and second stretch invariants, $J$ is the volumetric stretch (or third stretch invariant), $\mathrm{C}_{10}(\mathrm{MPa})$, $\mathrm{C}_{01}(\mathrm{MPa})$ and $\mathrm{D}_{1}$ are model parameters with values given in Table 1[11]
Table 1: Hyper elastic values of polyurethane

\begin{tabular}{|c|c|c|c|c|}
\hline Material & $\rho\left(\mathrm{kg} / \mathrm{mm}^{3}\right)$ & $\mathrm{C}_{10}$ & $\mathrm{C}_{01}$ & $\mathrm{D}_{1}$ \\
\hline Polyurethane & $1.07 \mathrm{E}^{-6}$ & 1.03 & 3.69 & 0 \\
\hline
\end{tabular}

Artery was modeled as quarter of a cylinder with length $10 \mathrm{~mm}$, inner diameter $3.50 \mathrm{~mm}$ and outer diameter $4 \mathrm{~mm}$. The inner surface of the artery was on the outer surface of the plaque. The outer surface of the Plaque was also cylindrical and inner surface with irregular shape to resemble with a blocked artery. The material of the artery was assumed to be a hyper elastic anisotropic material with third order Ogden strain energy function as given below (2).

$W=\sum_{i=1}^{a} \frac{2 u i \tilde{i}}{\alpha i^{2}}\left(\lambda_{1}{ }^{\alpha i}+\lambda_{2}{ }^{\alpha i}+\lambda_{a}{ }^{\alpha i}\right)+\sum_{i=1}^{a} \frac{1}{D_{i}}(J-1)^{2 i}$

Where $\lambda_{1}, \lambda_{2}, \lambda_{3}$ are the first, second and third stretch invariants, $\mathrm{J}$ is the volumetric stretch (or third stretch invariant) . $\mu_{\mathrm{i}}, \alpha_{\mathrm{i}}$ and $\mathrm{D}_{\mathrm{i}}$ are model parameters with values given in Table 2 .

Table 2: Material properties of artery

\begin{tabular}{|c|c|c|c|c|c|c|c|}
\hline$\rho\left(\mathrm{kg} / \mathrm{mm}^{3}\right)$ & $\mu_{1}$ & $\mu_{2}$ & $\mu_{3}$ & $\alpha_{1}$ & $\alpha_{2}$ & $\alpha_{3}$ & $\mathrm{D}_{1}$ \\
\hline $1.07 \mathrm{e}^{-6}$ & -7.04 & 4.23 & 2.85 & 24.48 & 25.00 & 23.54 & $8.95 \mathrm{e}^{-7}$ \\
\hline
\end{tabular}

The plaque of the diseased portion of artery was considered as bio material having anisotropic properties. It was also defined by hyper elastic Holzapfel strain energy function (3) and its properties are given in Table 3 . The quarter model of balloon, stent, plaque and artery assembly is shown in Fig. 2

$$
\Psi=c_{1}\left(I_{1}-3\right)+\frac{k_{1}}{2 k_{2}} \sum_{i=4,6}\left\{\exp \left[k_{2}\left(I_{i}-1\right)^{2}\right]-1\right\}
$$

Table 3: Material properties of plaque

\begin{tabular}{|c|c|c|c|}
\hline Material & $\left.\begin{array}{c}\text { Density } \\
\left(\mathrm{g} / \mathrm{cm}^{3}\right)\end{array}\right)$ & $\begin{array}{c}\text { Young's } \\
\text { Modulus } \\
(\mathrm{MPa})\end{array}$ & $\begin{array}{c}\text { Poisson's } \\
\text { ratio }\end{array}$ \\
\hline Plaque & 1.100 & 11.9 & 0.30 \\
\hline
\end{tabular}

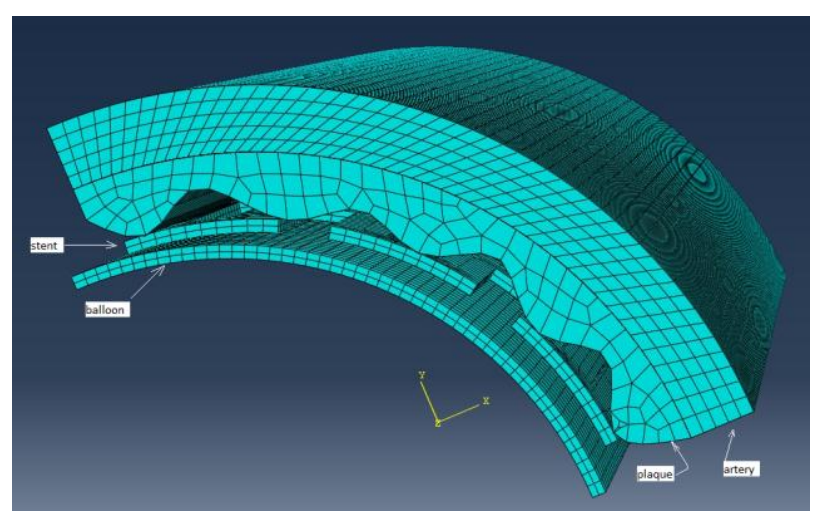

Fig. 2: 3D Meshed model (quarter) of balloon, stent and artery assembly 


\section{FE ANALYSIS}

The stent was expanded using a non compliant balloon by applying a pressure on the inner surface of the balloon. Three models were considered for the analysis. First model was only stent and it was expanded with different pressures ranges from $0.40 \mathrm{MPa}$ to $1 \mathrm{MPa}$. The second model was stent with balloon and it was also expanded by applying pressures on the inner surface of the balloon as done in the first model and the third model was a stent within a stenosed artery. In all these models a ramp up pressure was applied within a short period of $1.635 \mathrm{~s}$ with an initial increment of $0.1 \mathrm{~s}$ to expand the stent. Simulation time was set as small as possible in order to reduce the computational time. This is much shorter than the actual time required to expand the stent, but the kinetic energy was checked throughout the entire analysis to ensure that it was negligible compared to the internal energy. To get the expansion only in radial direction, symmetric boundary conditions were imposed on nodes of the stent in the plane of symmetry. Hence the nodes perpendicular to $\mathrm{X}$-axis is not allowed to move in $\mathrm{X}$ direction and all nodes perpendicular to $\mathrm{Y}$ - axis were not allowed to move in $\mathrm{Y}$ direction. No constrains were imposed along the Zaxis of the stent so that it can move freely in the axial direction during expansion. Fully integrated elements were used in the model, because reduced integration elements are prone to hourglass deformation. To analyze the stent, it was discretized by approximate global size of 0.055 which is proved as compatible mesh size for this particular model. 8 noded brick element with a total of 1347 elements was used. Material non linearity was accounted and a static general step with non linear geometry was used for analysis preprocessing.

In the second model, balloon and stent were assembled such that the outer surface of the balloon and the inner surface of the stent were in surface to surface contact. The outer surface of the balloon was taken as master surface and the inner surface of the stent as slave surface and a penalty frictional formulation with a frictional coefficient of 0.25 as interaction property. The loading and boundary condition for the stent were same as that of the first model. The balloon was assumed as fully tethered at both ends so that the expansion along the radial direction only permitted. Mesh control was implemented for both stent and balloon. The third phase of analysis was expansion of stent inside a diseased artery with same loading and boundary conditions to study the variation of stress and expansion of stented artery. Only quarter of the artery was considered for the assembly as shown in Fig.2. Artery was assembled on the outer surface of the stent by taking stent surface as master surface, inner surface of the artery as slave surface and a penalty frictional formulation with 0.05 as frictional coefficient. The boundary and loading conditions were same as the previous models and symmetric boundary condition were imposed on two ends of artery such that no displacement along $\mathrm{Z}$ direction. Mesh element and its size were same as balloon. A static general step with a non linear geometry was used to analyze the expansion of stent along with balloon and artery.

\section{RESULTS AND DISCUSSIONS}

In the first model as the stent expands, its geometry changes and the rectangular slot of the stent transforms to rhomboid shape. The stent deforms permanently when the applied pressure exceeds the yield strength of the material. However, the stress in some areas of the stent was typically high especially at the four corners of cells (slots). This is due to pulling of struts from each other to form a rhomboid shape. The shape of the stent after expansion and its stress - strain distribution are shown in Fig.3a and Fig.3b. Maximum von Mises stress and strain obtained at an inflating pressure of $0.40 \mathrm{MPa}$ was $0.236 \mathrm{MPa}$ and $0.809 \mathrm{~mm}$ respectively. In comparison with $0.249 \mathrm{MPa}$, obtained from similar analysis [11]. This is within acceptable limit and hence the methodology of this analysis has been validated.

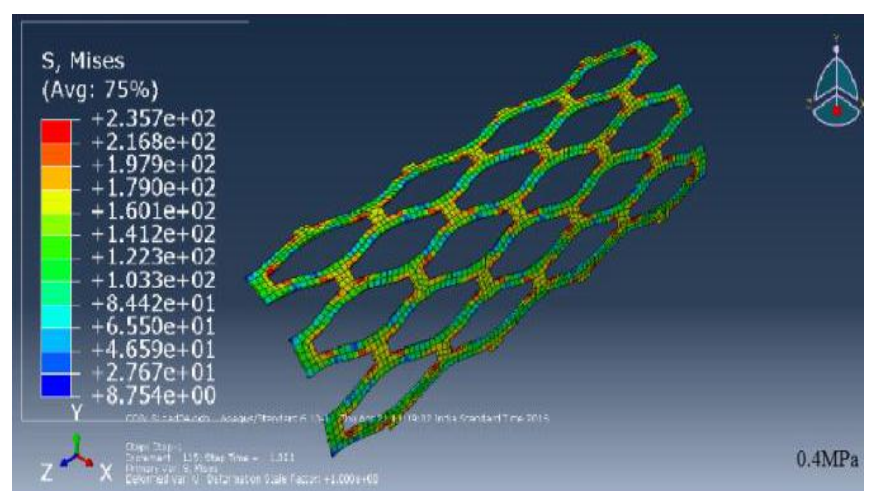

Fig. 3a: Stress distribution on stent at $0.40 \mathrm{MPa}$ (only stent)

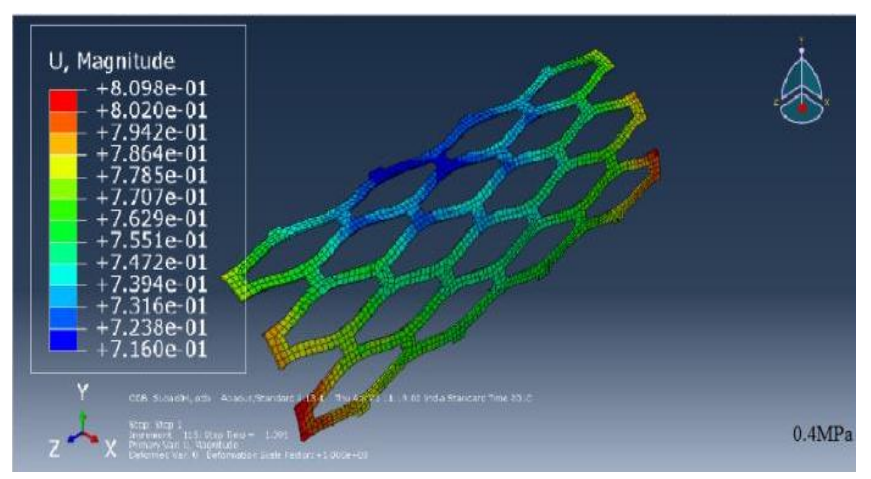

Fig.3b: Elemental strain on stent at $0.40 \mathrm{MPa}$ (only stent)

In the second phase, the balloon stent model was expanded using a balloon and the ends of the balloon were constrained so that no displacement at the balloon ends in any direction. Maximum von Mises stress of $0.22 \mathrm{MPa}$ and maximum displacement of $0.59 \mathrm{~mm}$ was observed on stent at the end of expansion and it was at the four corners of cell at the middle of the stent. Also the expansion was found more uniform and the stresses are evenly distributed. Since the balloon was encastered on both ends, its expansion at the middle found larger than both the ends. 


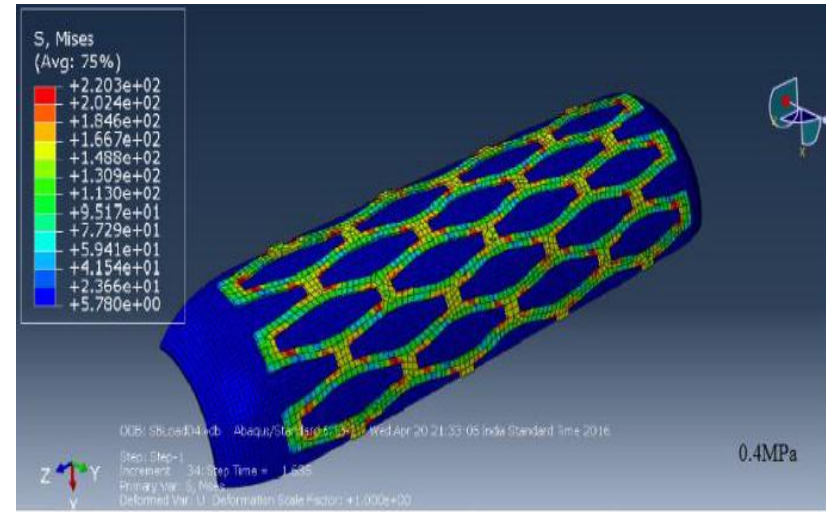

Fig.4a: Stress distribution on stent at $0.40 \mathrm{MPa}$ (stent with balloon)

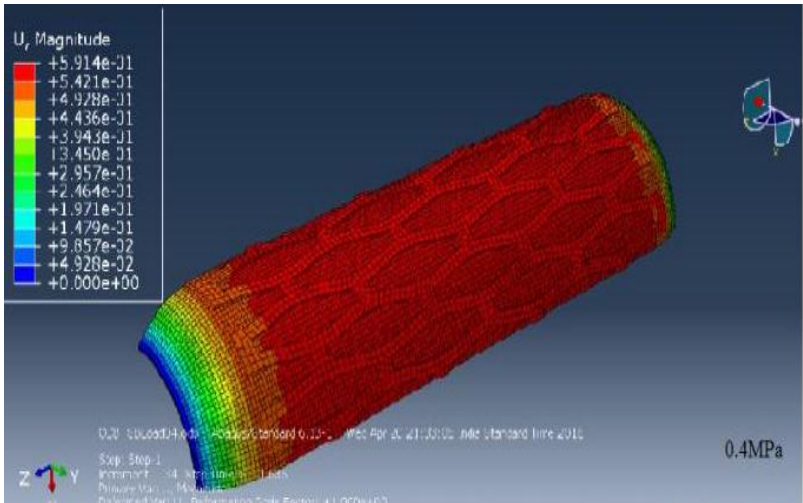

Fig. 4b: Elemental strain on stent at $0.40 \mathrm{MPa}$ (stent with balloon)
The stress distribution on the stent at a pressure of $0.40 \mathrm{MPa}$ is shown in Fig. 4a and the displacement is shown in Fig. 4b. Maximum von Mises stress of $0.223 \mathrm{MPa}$ and maximum displacement of 0.59 was observed on the stent. In the third phase of the simulation, the expansion of stent within a diseased artery was performed with variable pressures. Since the artery was having a frictional contact with the stent, the stress induces on the artery by the stent expands. Finally the plaque is compressed and the opening of the stenosed artery is increased. However due to the resistance from artery and plaque more pressure is required to expand the stent. Therefore for a same pressure, the stress and deformation on the stent inside the artery is less compared to that of a plain stent. Maximum stress of $0.210 \mathrm{MPa}$ and displacement of $0.435 \mathrm{~mm}$ was observed on the stent at a pressure of $0.4 \mathrm{MPa}$. Maximum stress and displacement on the stent with different pressures for three models are shown in Table 4. It was observed that as the inflating pressure increases the stress and strain on stent increases in the entire model. As the surface area of the balloon is larger than stent, the force exerted on the stent with balloon is quite large and hence expansion as well as stress is higher on balloon stent model. The characteristics of balloon also influence the expansion of stent so that a non compliant balloon can expand more than a compliant balloon.

Table 4: Stress-strain variation with pressure in different models

\begin{tabular}{|c|c|c|c|c|c|c|}
\hline \multirow{2}{*}{$\begin{array}{c}\text { Pressure } \\
(\mathrm{MPa})\end{array}$} & \multicolumn{2}{|c|}{$\begin{array}{c}\text { Model 1 } \\
\text { (only stent) }\end{array}$} & \multicolumn{2}{c|}{ Model 2 (stent with balloon) } & \multicolumn{2}{c|}{ Model 3 (stent,balloon and artery) } \\
\cline { 2 - 7 } & Stress (MPa) & Strain (mm) & Stress (MPa) & Strain (mm) & Stress (MPa) & Strain (mm) \\
\hline 0.4 & 0.236 & 0.81 & 0.223 & 0.591 & 0.210 & 0.435 \\
\hline 0.6 & 0.245 & 1.06 & 0.253 & 1.230 & 0.221 & 0.622 \\
\hline 0.8 & 0.260 & 1.44 & 0.278 & 1.971 & 0.226 & 0.742 \\
\hline 1.0 & 0.277 & 1.95 & 0.301 & 2.400 & 0.229 & 0.835 \\
\hline
\end{tabular}

The variation of stress and strain with respect to inflating pressure for three different models are graphically shown in Fig. 5a and Fig. 5b . Stent within artery gives less expansion and less stress and stent with balloon gives high expansion and high stress. Compared to the expansion of plain stent, a reduction of $10 \%$ in the stress and $45 \%$ in strain was noticed as stent expands inside the stenosed artery. The induced stress on artery is only $50 \%$ of the stress on the stent but the strain on artery is $85 \%$ of the stent. Therefore it is evident that the inflating pressure required for a specific opening of a stenosed artery is higher than that of a stent without artery and the pressure is determined by the characteristics of artery as well as plaque and the percentage of block . 


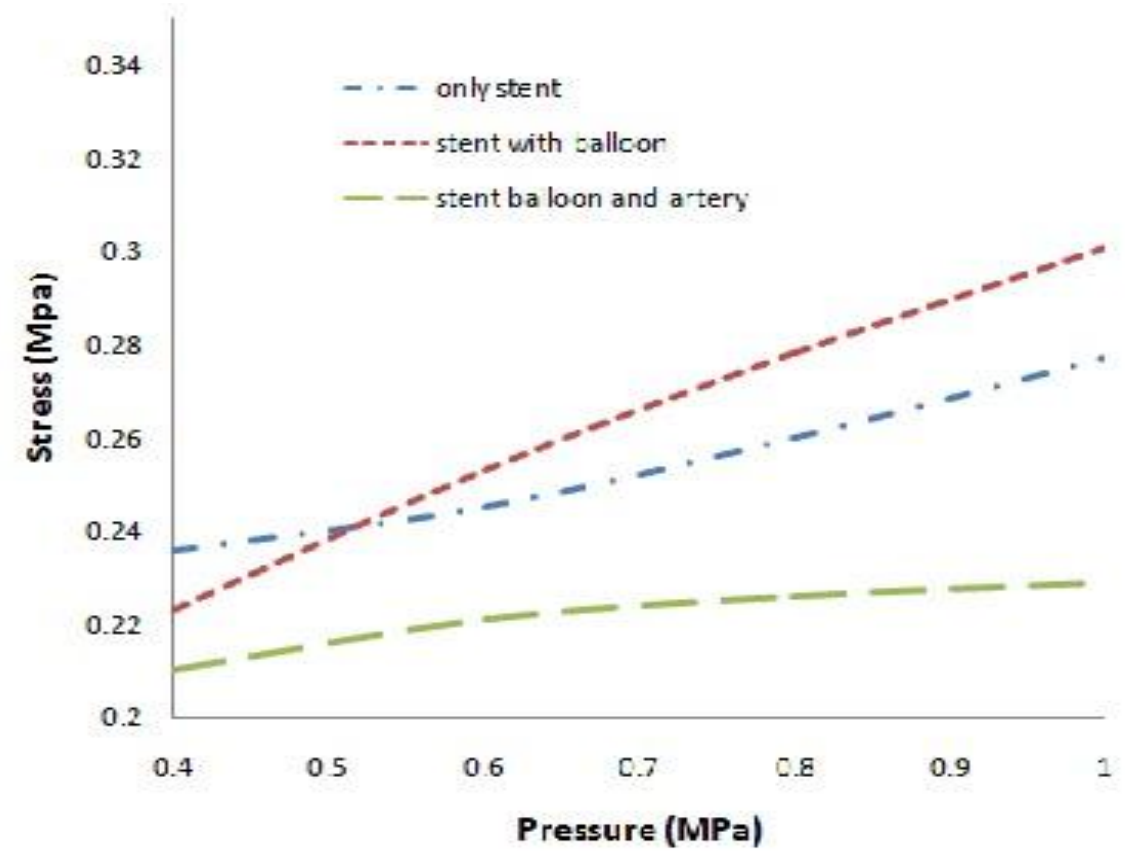

Fig.5a: Maximum stress at various pressures

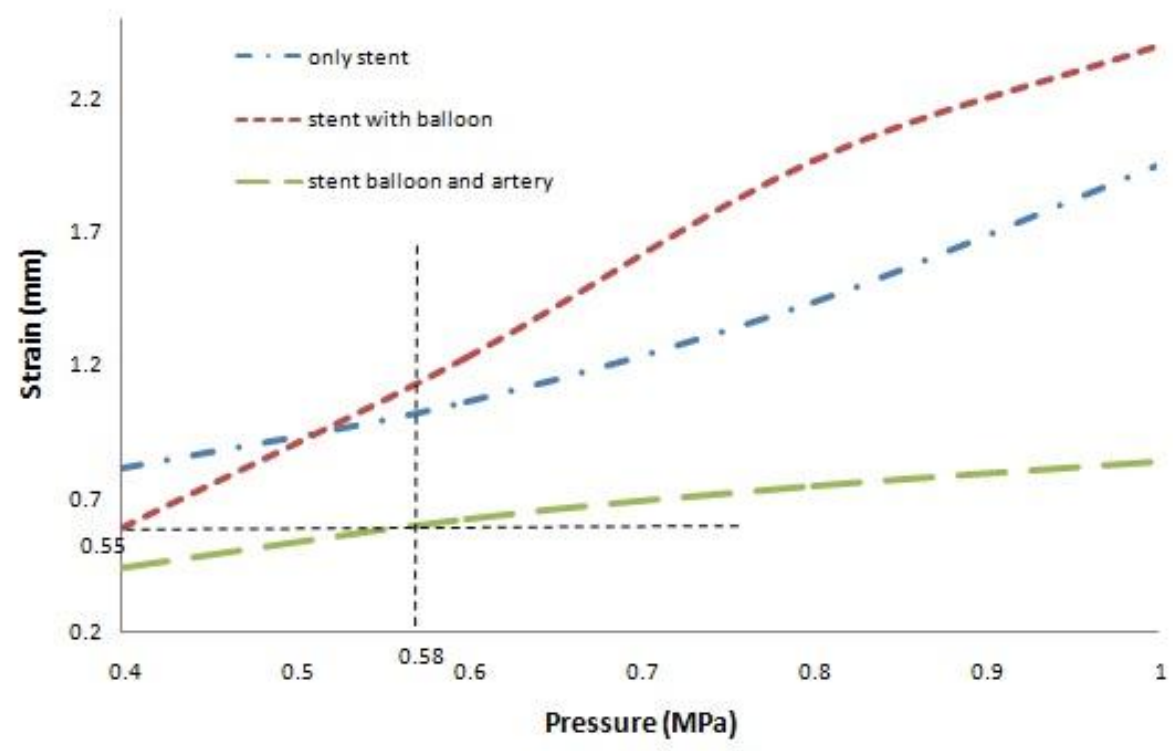

Fig. 5b: Maximum expansion at various pressures

\section{Optimum inflating pressure for a stenosed artery}

The main objective of this paper was to find out the optimum pressure required for recovering the original diameter a diseased artery. A diseased artery of $4 \mathrm{~mm}$ diameter with a plaque of $0.25 \mathrm{~mm}$ thickness $\quad(20.42 \%$ block $)$ was considered for this study. Diameter of stent before expansion was $2.9 \mathrm{~mm}$. Hence the stent has to expand to a radial displacement of $0.55 \mathrm{~mm}$ to achieve the original diameter of the artery. The exact pressure required for this expansion was found from the graph shown in Fig. 5b. For the stent within artery a displacement of $0.55 \mathrm{~mm}$ was obtained for an inflating pressure of $0.58 \mathrm{MPa}$. This has been cross verified by conducting a simulation of expansion of a stenosed artery with an inflating pressure of $0.58 \mathrm{MPa}$ and the same displacement of $0.55 \mathrm{~mm}$ and maximum stress of $0.218 \mathrm{MPa}$ was obtained as shown in Fig. 6a and Fig. $6 b$. 


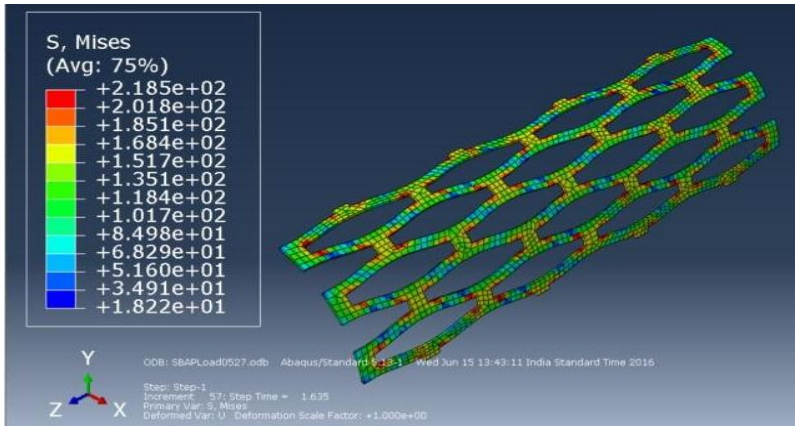

Fig. 6a: Stress distribution on stent at $0.58 \mathrm{MPa}$ in a stenosed artery

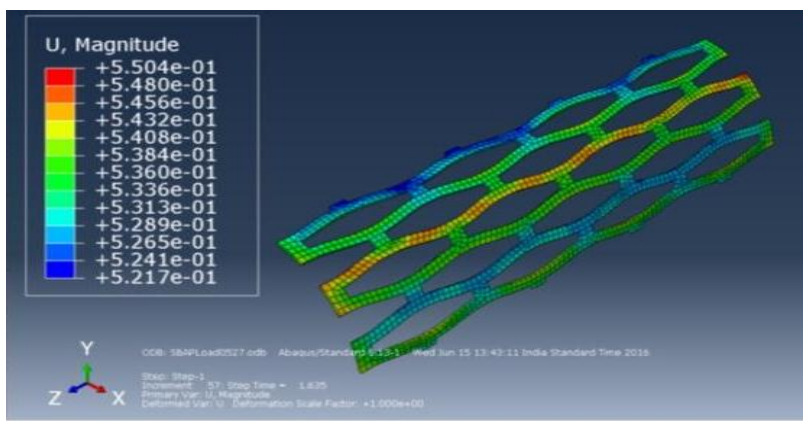

Fig. 6b: Strain on stent at $0.58 \mathrm{MPa}$ in stenosed artery

\section{CONCLUSIONS}

The expansion characteristics of stent, stent with balloon and stent within artery, were compared using FE analysis. The stress distribution and expansion of stent were analyzed with variable inflating pressures. It was found that stress concentration increases with inflating pressure and the expansion of stent was more uniform and larger when it was expanded with balloon. Maximum stress was found on the four corners of the slots of the middle of the stent in all the three models. Stress and strain on the artery were due to the impact of stent and it was significantly low compared to stent. The expansion of artery with respect to stent depends on the characteristics of plaque and percentage of block. It implies that more pressure is required to expand an artery with high level of block. In this study exact pressure for a stenosed artery with $20.42 \%$ of block was found as $0.58 \mathrm{MPa}$ by graphical method and was cross verified by simulation. It can be concluded that the expansion characteristics of stent in a real situation is different from the expansion of plain stent and is difficult to assess the optimum pressure for a stent deployment in a stenosed artery. The above methodology can be utilized for predicting the exact inflating pressure for a stent deployment in a stenosed artery. This is a preliminary work to develop an aiding tool for predicting the exact pressure before performing angeoplasty. In this analysis only inflation of the balloon was considered but in actual practice due to elastic recoil, stent will experience a small reduction in the diameter after balloon deflation. This can also be considered for further investigation to get more realistic results.

\section{REFERENCES}

[1] Puranik AS, Dawson ER, Peppas NA, "Recent advances in drug eluting stents", Int. Journal Pharm, 79, 441-665,2013.

[2] Hashim V., Dileep P.N. Srinath P, Rahul NR, Mufaz FB, Ajoy U., " Computational modelling and analysis to investigate and compare the mechanical behaviour of various stent designs using finite element methods", International journal of Current Engineering and Scientific Research, Vol.4 Issue 9. 2017.

[3] Frederique Etava, Gerard Finet, et.al. "Mechanical properties of coronary stents determined by using FEA.," Journal of Biomechanics 34 (2001) 1065-1075.

[4] Hyre MR, Pulliam RM, Squire JG. "Prediction of stent endflare, arterial stresses and flow patterns in a stenotic artery", Virginia military institute Journals, 2, 300-308, 2009.

[5] Francesco Migliavacca, Lorenza Petrini, Maurizio Colombo, Ferdinando Auricchio, Riccardo Pietrabissa., "Mechanical behavior of coronary stents investigated through the finite element method", Journal of Biomechanics, 35, 803-811, 2002.

[6] Hao-Ming Hsiao, Yi-Hsiang Chiu, et.al.," Computational modelling of effects of intra vascular stent design on key mechanical and hemodynamic behaviour," Computer Aided Design 44(2012) 757-765.

[7] S.N. David Chua, B.J. Mac Donald, M.S.J. Hashmi., "Effects of varying slotted tube (stent) geometry on its expansion behaviour using finite element method", Journal of Materials Processing Technology, 1764- 1771, 2004.

[8] N. Eshghi, M. H. Hojjati, M. Imani, A. M. Goudarzi., "Finite element analysis of mechanical behaviors of coronary stent", Procedia Engineering, 10, 3056-3061, 2011.

[9] Jakub Bukala, Piotr Kwiatkowski, Jerzy Malachowski., "Numerical analysis of stent expansion process in coronary artery stenosis with the use of non-compliant balloon", Bio cybernetics and biomedical engineering, 97,145-156, 2016

[10] Houman Zahedmanesh, Daniel John Kelly, Caitriona Lally., "Simulation of a balloon expandable stent in a realistic coronary artery - Determination of the optimum modelling strategy", Journal of Biomechanics, 43, 2126-2132, 2010.

[11] S.N. David Chua, B.J. Mac Donald, M.S.J. Hashmi., "Finite element simulation of stent and balloon interaction", Journal of Materials Processing Technology 143-144 (2003) 591-597 